\title{
Haptic Feedback for Passengers Using Public Transport
}

\author{
Ricky Jacob, Bashir Shalaik, Adam C. Winstanley, and Peter Mooney \\ Department of Computer Science, National University of Ireland, \\ Maynooth Co. Kildare, Ireland \\ \{rjacob, bsalaik, adamw\} ecs. nuim.ie
}

\begin{abstract}
People using public transport systems need two kinds of basic information - (1) when, where and which bus/train to board, and (2) when to exit the vehicle. In this paper we propose a system that helps the user know his/her stop is nearing. The main objective of our system is to overcome the 'neck down' approach of any visual interface which requires the user to look into the mobile screen for alerts. Haptic feedback is becoming a popular feedback mode for navigation and routing applications. Here we discuss the integration of haptics into public transport systems. Our system provides information about time and distance to the destination bus stop and uses haptic feedback in the form of the vibration alarm present in the phone to alert the user when the desired stop is being approached. The key outcome of this research is haptics being an effective alternative to provide feedback for public transport users.
\end{abstract}

Keywords: haptic, public transport, real-time data, gps.

\section{Introduction}

Haptic technology, or haptics, is a tactile feedback technology that takes advantage of our sense of touch by applying forces, vibrations, and/or motions to the user through a device. From computer games to virtual reality environments, haptics has been used for a long time [8]. One of the most popular uses is the Nintendo Wii controllers which give the user forced feedback while playing games. Some touch screen phones have integrated forced feedback to represent key clicks on screen using vibration alarm present on the phone. Research into the use of the sense of touch to transfer information has been going on for years. Van Erp, who has been working with haptics for over a decade, discusses the use of the tactile sense to supplement visual information in relation to navigating and orientating in a Virtual Environment [8]. Jacob et al [11] provided a summary of the different uses of haptics and how it is being integrated into GIS. Hoggan and Brewster [10] feel that with the integration of various sensors on a smartphone, it makes it an easier task to develop simple but effective communication techniques on a portable device. Heikkinen et al [9] states that our human "sense of touch is highly spatial and, by its nature, tactile sense depends on the physical contact to an object or its surroundings". With the emergence of smart phones that come enabled with various sensors like accelerometer, magnetometer, gyroscope, compass and GPS, it is possible to develop applications that provide navigation information in the form of haptic feedback [11] [13]. The "PocketNavigator" 
application which makes use of the GPS and compass helps the user navigate by providing different patterns of vibration feedback to represent various directions in motion. Jacob et al [12] describe a system which integrates OpenStreetMap data, Cloudmade Routing API [21], and pedestrian navigation and provides navigation cues using haptic feedback by making use of the vibration alarm in the phone. Pedestrian navigation using bearing-based haptic feedback is used to guide users in the general direction of their destination via vibrations [14]. The sense of touch is an integral part of our sensory system. Touch is also important in communicating as it can convey non-verbal information [9]. Haptic feedback as a means for providing navigation assistance to visually impaired have been an area of research over the past few years. Zelek augments the white cane and dog by developing this tactile glove which can be used to help a visually impaired user navigate [15].

The two kinds of information that people using public transport need are - (1) when, where and which bus/train to board, and (2) when to exit the vehicle to get off at the stop the user needs to go to. Dziekan and Kottenhoff [7] study the various benefits of dynamic real-time at-stop bus information system for passengers using public transport. The various benefits include - reduced wait time, increased ease-of use and a greater feeling of security, and higher customer satisfaction. The results of the study by Caufiled and O'Mahony demonstrate that passengers derive the greatest benefit from accessing transit stop information from real-time information displays [16]. The literature states that one of the main reasons individuals access real-time information is to remove the uncertainty when using public transit. Rehrl et al [17] discusses the need for personalized multimodal journey planners for the user who uses various modes of transport. Koskinen and Virtanen [18] discuss information needs from a point of view of the visually impaired in using public transport real time information in personal navigation systems. Three cases presented are: (1) using bus real time information to help the visually impaired to get in and leave a bus at the right stop, (2) boarding a train and (3) following a flight status. Bertolotto et al [4] describe a BusCatcher system. The main functionality provided include: display of maps, with overlaid route plotting, user and bus location, and display of bus timetables and arrival times. Turunen et al [20] present approaches for mobile public transport information services such as route guidance and push timetables using speech based feedback. Bantre et al [2] describes an application called "UbiBus" which is used to help blind or visually impaired people to take public transport. This system allows the user to request in advance the bus of his choice to stop, and to be alerted when the right bus has arrived. An RFID based ticketing system provides the user's destination and then text messages are sent by the system to guide the user in real time [1]. The Mobility-for-All project identifies the needs of users with cognitive disabilities who learn and use public transportation systems [5]. They present a sociotechnical architecture that has three components: a) a personal travel assistant that uses real-time Global Positioning Systems data from the bus fleet to deliver just-intime prompts; b) a mobile prompting client and a prompting script configuration tool for caregivers; and c) a monitoring system that collects real-time task status from the mobile client and alerts the support community of potential problems. There is mention about problems such as people falling asleep or buses not running on time 